sation. These concluding chapters stamp Mr. Galton as an original thinker, as well as a forcible and eloquent writer; and his book will take rank as an important and valuable addition to the science of human nature.

ALFRED R. WALLACE

\section{SPECTRUM ANALYSIS}

Die Spectral Anclyse in ihrer Anqeendung auf die Stoffe der Erde und die Nutur der Fimmelskörper. By Lr. H. Schellen, director der Realschule 1.0., Cologne. (Brunswick, Westermann, 1870. London: Williams and Norgate.)

TH $\mathrm{iS}$ book contains an accurate and luminous account of the recent discoveries in celestial chemistry and physics, and especially of the researches of our countrymen Huggins and Lockyer. As regards the completeness of that portion of the work bearing directly upon terrestrial chemistry, readers will, I fear, be disappointed. The first division of the book is devoted to a description of the means employed for the artificial evolution of light and heat of great intensity, beginning with combustions in oxygen, and ending with the electric-light. The second division is headed "The simple and compound spectra in their application to terrestrial matter;" whilst in the third and most important division Schellen considers the application of spectrum analysis to the heavenly bodies. The illustrations throughout the work are good, though many of them are not new, and are borrowed, without acknowledgment, from other books.

With respect to the physical constitution of the sun, it benoves us in this, the infancy of our knowledge, to be very careful in trawing positive conclusions. In the first place, there is no doubt that whilst Kirchhoff's original theory must undergo certain modifications, it will remain in its grand features as having first pointed out to us the true physical condition of the sun. The discovery of the chromosphere by $\mathrm{Mr}$. Lockyer, in which, as a rule, only the bright hydrogen lines are seen, together with the yellow mysterious line of unknown origin, renders it difficult for us, especially if we accept Frankland and Lockyer's conclusions respecting the excessive tenuity of the upper chromospheric layers, to suppose that an atmosphere containing iron and the other 13 difficultly volatilisable metals can exist outside the chromosphere of sufficient density to effect such a powerful selective absorption as we see in the darkness of Fraunhofer's lines. Hence we should be inclined to agree with Lockyer that the absorption does not take place, as Kirchhoff suggested, in a far outlying layer of solar atmosphere, or in what we term the corona, but that the dark lines are produced within the chromosphere. But, on the ofher hand, upon what known physical basis are we entitled to assume that the higher lying portions of the solar atmosphere consist almost entirely of glowing hydrogen gas, whilst the lower lying layers contain the more easily condensible gases of the other 14 elements? The well-known laws of gaseous diffusion (to say nothing of the cyclones of vast magnitude and of enormous rapidity, which Lockyer has taught us are constantly mingling up the various layers of solar atmosphere), forbid us to suppose that the lighter hydrogen gas can ascend whilst the heavier metallic gases remain quietly below. If the components of the solar atmosphere are gaseous, they must be uniformly, or nearly uniformly, mixed. How then can we account for the constant presence in the chromosphere of the hydrogen lines, whereas the lines of the other constituents of the solar atmosphere are scarcely visible, except in special cases of the accasional projection of the vapours of magnesium and other metals, whilst the absorption is to occur in a lower gaseous layer, having a totally different composition?

Another point to be remembered is, that according to the law of exchanges the fact of the existence of absorption necessitates the existence of a lower temperature in the absorptive medium than in the media (either above or below) in which such absorption is not ex. hibited, and which may either give continuous or broken spectra, according to the physical and chemical nature of the incandescent bodies. How then can the iron and magnesium vapour exist nearer to the white-hot body of the sun than the hydrogen and yet possess a lower temperature? I am here forcibly. reminded of the plausibility of a suggestion thrown out by Kirchhoff, in a conversation with me a few weeks ago, viz. : that the upper regions of the solar atmosphere may be constantly illumed by discharges of electricity; that the incandescent hydrogen may be heated not from below but from within its own mass, either by continuous flashes of lightning or constant auroral discharges ; and, indeed, Zöllner has noticed the flashing out of certain bright points in the prominences, which may possibly be caused by solar lightning.

We must also bear in mind that the existence in the sun of a solid or liquid white-hot nucleus, as originally assumed, is not proved by the resuits of subsequent research; inasmuch as we learn from the recent researches of Frankland, Lockyer, and Wüllner (as indeed we may do from much older experiments), that incandescent gases under certain physical conditions emit white light and yield a continuous spectrum. So that spectrum analysis does not give us any certain information as to the physical state of that portion of the sun's body from which the main portion of light and heat proceeds.

H. E. ROSCOE

\section{OUR BOOK SHELF}

Essays on Physiological Subjects. By Gilbert W. Child, M.A., F.L.S., F.C.S. Second edition, with Additions; pp. 293. (London: Longmans, Green, and Co. 186.) THE present edition of Dr. Child's work is by no means a mere reprint of the last. It has undergone considerable modifications, chiefly in the form of additions, which will tend to make it more acceptable to a large class of readers. There is an almost entirely new essay on "Some Aspects of the Theory of Evolution," in which he endeavours to show how this theory is related to religious belief. $\mathrm{He}$ believes its proper meaning and tendency to have been much misunderstood; that far from being an "atheistical" conception, it is in reality only the scientific form of natural religion. The subject of "Physiological Experimentation on Animals" is also considered, whilst the last and longest essay, also new, is entitled "Physiological Psychology," in which he endeavours to make known to persons whose chief interest is in psychological rather than physiological science, all the chief points in the anatomy of the nervous system, necessary to be understood before he could explain, as he also attempts to do, the principal physiological conclusions which have been arrived at concerning brain action and mind. 